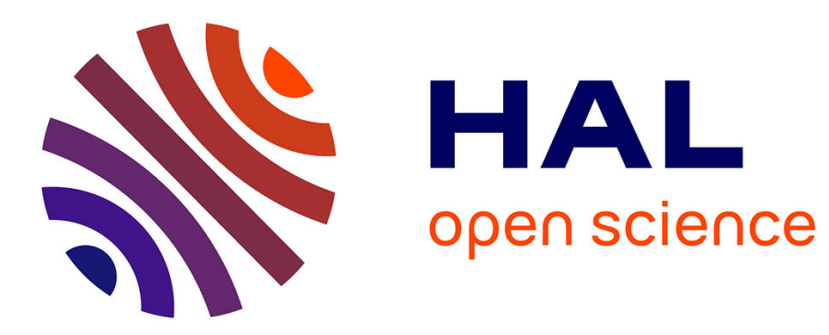

\title{
Hybrid Regional Stabilization of Linear Systems with Actuator Saturation and Multi-Rate Samplers
}

Francesco Ferrante, Ricardo Sanfelice, Sophie Tarbouriech

\section{To cite this version:}

Francesco Ferrante, Ricardo Sanfelice, Sophie Tarbouriech. Hybrid Regional Stabilization of Linear Systems with Actuator Saturation and Multi-Rate Samplers. ECC 2018 - 16th European Control Conference, Jun 2018, Limassol, Cyprus. pp.1-6, 10.23919/ECC.2018.8550561 . hal-01721712

\section{HAL Id: hal-01721712 \\ https://hal.science/hal-01721712}

Submitted on 6 Jul 2020

HAL is a multi-disciplinary open access archive for the deposit and dissemination of scientific research documents, whether they are published or not. The documents may come from teaching and research institutions in France or abroad, or from public or private research centers.
L'archive ouverte pluridisciplinaire HAL, est destinée au dépôt et à la diffusion de documents scientifiques de niveau recherche, publiés ou non, émanant des établissements d'enseignement et de recherche français ou étrangers, des laboratoires publics ou privés. 


\title{
Hybrid Regional Stabilization of Linear Systems with Actuator Saturation and Multi-Rate Samplers
}

\author{
Francesco Ferrante, Ricardo G. Sanfelice, and Sophie Tarbouriech
}

\begin{abstract}
Regional stability analysis of linear systems with multi-rate samplers and actuator saturation is studied. A hybrid controller is used to perform a fusion of measurements sampled at different times. In between sampling events, the controller behaves as a copy of the plant. When a new measurement is available, the controller state undergoes a jump. The resulting system is analyzed in a hybrid system framework. Sufficient conditions in the form of matrix inequalities are given to determine estimates of the basin of attraction of the closed-loop system. Finally, the effectiveness of the proposed methodology is shown in an example.
\end{abstract}

\section{INTRODUCTION}

Control of sampled-data systems has been attracting the attention of researchers for a long time and its interest is still vivid in the community as pointed out by the recent survey [1]. Capturing the effect of sampled-data information in control loops is indeed fundamental in addressing the challenges posed by modern engineered systems in which the use of embedded systems and communication networks is central. One of the most investigated aspects in the literature of sampled-data systems pertains to the study of aperiodic sampled-data systems, that is systems in which the exchange of information from the plant to the controller happens in an intermittent aperiodic fashion; see for example [2], [3], just to cite a few. In fact, aperiodic sampled-data systems may be used to capture different phenomena occurring in data networks as dropouts, sampling jitter, etc; see [1].

A common assumption in sampled-data control consists of considering synchronous sampling of different variables. However, in certain applications due to technological constraints, some variables may happen to be measured with a different sampling-rate than others, making the assumption of synchronous sampling restrictive; see, e.g., [4]. This need promoted the development of new tools devoted to the analysis of multi-rate control systems; see [5], [6].

Francesco Ferrante is with Univ. Grenoble Alpes, CNRS, Grenoble INP, GIPSA-lab, 38000 Grenoble, France. Ricardo G. Sanfelice is with Department of Computer Engineering, University of California, Santa Cruz, CA 95064. Sophie Tarbouriech is with LAAS-CNRS, Université de Toulouse, CNRS, Toulouse Email: francesco.ferrante@gipsa-lab.fr, ricardo@ucsc.edu, tarbour@laas.fr

This work has been partially developed when the first author was with Department of Computer Engineering, University of California, Santa Cruz. This research has been partially supported by the National Science Foundation under CAREER Grant no. ECS-1450484, Grant no. ECS1710621, and Grant no. CNS-1544396, by the Air Force Office of Scientific Research under Grant no. FA9550-16-1-0015, by the Air Force Research Laboratory under Grant no. FA9453-16-1-0053, and by CITRIS and the Banatao Institute at the University of California. This version fixes some typographical errors/undefined symbols in the published paper. Footnotes in red indicate the corrections. Last update July 6, 2020.
Another limitation that is naturally present in actual control systems concerns to actuator saturation. The study of saturated control systems is by now a very mature research area as testified by the large amount of publications in this field [7], [8]. Many approaches have been proposed in the literature to analyze the effect of actuator saturation in (single-rate) sampled-data control systems; see [9], [10], [11]. However, to the best of our knowledge, no results are found in the literature to deal with actuator saturation in a multi-rate setting.

To fill this gap, in this paper, building on the modelbased controller architecture presented in [12], originally proposed for single-rate non-saturated linear systems, we propose sufficient conditions for regional stability analysis of linear systems in the presence of actuator saturation and multi-rate samplers. The approach we propose is enabled by the hybrid systems framework in [13]. Sufficient conditions in the form of matrix inequalities are given to characterize estimates of the basin of attraction of the closed-loop system. Semidefinite programming is used to reduce the conservatism of such estimates.

The remainder of this paper is structured as follows. Section I-A presents some preliminaries on hybrid systems. Section II presents the problem we solve and the adopted modeling approach. Section III is dedicated to the main results. Section IV shows the effectiveness of the proposed approach in a numerical example. Due to space constraints proofs will be published elsewhere.

Notation: The set $\mathbb{N}$ is the set of positive integers including zero, the set $\mathbb{N}_{>0}$ is the set of strictly positive integers, $\mathbb{R}_{\geq 0}$ represents the set of nonnegative real scalars, $\mathbb{R}^{n \times m}$ represents the set of the $n \times m$ real matrices, and $\mathcal{S}_{+}^{n}$ is the set of $n \times n$ symmetric positive definite matrices. The identity matrix is denoted by $\mathbf{I}$, whereas the null matrix is denoted by $\mathbf{0}$. For a matrix $A \in \mathbb{R}^{n \times m}, A^{\top}$ denotes the transpose of $A, A_{(i)}$ the $i$-th row of $A, A_{(i)}^{\top}$ the $i$-th row of $A^{\top}$, and $\operatorname{He}(A)=A+A^{\top}$. For two symmetric matrices, $A$ and $B, A>B, A \geq B$ mean that $A-B$ is, respectively, positive definite and positive semidefinite. In partitioned symmetric matrices, the symbol - stands for symmetric blocks. The matrix $\operatorname{diag}\left\{A_{1}, A_{2}, \ldots, A_{n}\right\}:=\bigoplus_{i=1}^{n} A_{n}$ is the block-diagonal matrix having $A_{1}, A_{2}, \ldots, A_{n}$ as diagonal blocks. For a vector $x \in \mathbb{R}^{n}$, $|x|$ denotes the Euclidean norm and for a matrix $A \in \mathbb{R}^{n \times n},|A|$ denotes the Euclidean induced-norm. Given two vectors $x, y$, we denote $(x, y)=\left[x^{\top} y^{\top}\right]^{\top}$. For two vectors $x, y \in \mathbb{R}^{n}, x \succeq y$ means that the components of $x-y$ are nonnegative. Given a vector $x \in \mathbb{R}^{n}$ and a closed set $\mathcal{A}$, the distance of $x$ to $\mathcal{A}$ is defined as $|x|_{\mathcal{A}}=\inf _{y \in \mathcal{A}}|x-y|$. For any function $z: \mathbb{R} \rightarrow \mathbb{R}^{n}$, 
we denote $z\left(t^{+}\right):=\lim _{s \rightarrow t^{+}} z(s)$. Given $P \in \mathcal{S}_{+}^{n}$ and $c>0$, then $\mathcal{E}(P, c):=\left\{x \in \mathbb{R}^{n}: x^{\top} P x \leq c\right\}$. Given a set $S$, we denote $\operatorname{co} S$ the convex-hull of $S$. The symbol $\times$ denotes the Cartesian products of two sets, while given sets $S_{1}, S_{2}, \ldots, S_{n}$, we denote $\mathrm{X}_{i=1}^{n} S_{i}=S_{1} \times S_{2} \times \cdots \times S_{n}$. Given two sets $X \subset \mathbb{R}^{n+m}$ and $Y \subset \mathbb{R}^{n}, \Pi_{Y}(X):=\left\{x \in Y: \exists y \in \mathbb{R}^{m}\right.$ s.t. $\left.(x, y) \in X\right\}$.

\section{A. Preliminaries on Hybrid Systems}

We consider hybrid systems with state $x \in \mathbb{R}^{n_{x}}$ of the form

$$
\mathcal{H}\left\{\begin{array}{cccc}
\dot{x} & =f(x) & & x \in \mathcal{C} \\
x^{+} & \in G(x) & & x \in \mathcal{D}
\end{array}\right.
$$

In particular, we denote the function $f: \mathbb{R}^{n_{x}} \rightarrow \mathbb{R}^{n_{x}}$ as the flow map, $\mathcal{C} \subset \mathbb{R}^{n_{x}}$ as the flow set, the set-valued map $G: \mathbb{R}^{n_{x}} \rightrightarrows \mathbb{R}^{n_{x}}$ as the jump map, and $\mathcal{D} \subset \mathbb{R}^{n_{x}}$ as the jump set.

A set $E \subset \mathbb{R}_{>0} \times \mathbb{N}$ is a hybrid time domain if it is the union of a finite or infinite sequence of intervals $\left[t_{j}, t_{j+1}\right] \times$ $\{j\}$, with the last interval (if existent) of the form $\left[t_{j}, T\right)$ with $T$ finite or $T=\infty$. Given a hybrid time domain $E$, we denote $\sup _{j} E=\sup \left\{j \in \mathbb{N}: \exists t \in \mathbb{R}_{\geq 0}\right.$ s.t. $\left.(t, j) \in E\right\}$.

A function $\phi: \operatorname{dom} \phi \rightarrow \mathbb{R}^{n_{x}}$ is a hybrid arc if: $\operatorname{dom} \phi$ is a hybrid-time domain and $\phi(\cdot, j)$ is locally absolutely continuous for each $j$. A hybrid arc $\phi$ is a solution to $\mathcal{H}$ if it satisfies the dynamics of $\mathcal{H}$; see [13] . A solution $\phi$ to $\mathcal{H}$ is maximal if it cannot be extended and is complete if $\operatorname{dom} \phi$ is unbounded. In particular, given $S \subset \mathbb{R}^{n_{x}}$, we denote $\mathcal{S}_{\mathcal{H}}(S)$ the set of maximal solutions $\phi$ to $\mathcal{H}$ with $\phi(0,0) \in S$, if $S$ is omitted, $\phi \in \mathcal{S}_{\mathcal{H}}$ means that $\phi$ is a maximal solution to $\mathcal{H}$; see [13] for more details.

\section{Problem Statement}

\section{A. System description}

We consider continuous-time linear time-invariant systems with saturated actuators of the form

$$
\mathcal{P}\left\{\begin{array}{c}
\dot{x}_{p}=A x_{p}+B u \\
u=\operatorname{sat}(v)
\end{array}\right.
$$

where $x_{p} \in \mathbb{R}^{n_{p}}$ is the plant state, $u \in \mathbb{R}^{n_{u}}$ is the plant input and $v \in \mathbb{R}^{n_{u}}$ is the actuator input. The function $v \mapsto \operatorname{sat}(v)$ is the symmetric decentralized saturation function with saturation levels $\bar{v}_{1}, \bar{v}_{2}, \ldots, \bar{v}_{n_{u}} \in \mathbb{R}_{>0}$, whose components for each $v \in \mathbb{R}^{n_{u}}$ are defined as

$\operatorname{sat}(v)_{i}=\operatorname{sat}\left(v_{i}\right):=\min \left(\left|v_{i}\right|, \bar{v}_{i}\right) \operatorname{sign}\left(v_{i}\right) \quad i=1,2, \ldots, n_{u}$

Moreover, $A$ and $B$ are constant matrices of appropriate dimensions.

As in [14], assume that sporadic and asynchronous measurements of the components of the plant state $x_{p}$ are available. More precisely, assume that the components of the plant state $x_{p}$ are clustered into $n_{s} \leq n_{p}$ subvectors $x_{p}^{(1)} \in \mathbb{R}^{n_{p_{1}}}, x_{p}^{(2)} \in \mathbb{R}^{n_{p_{2}}}, \ldots, x_{p}^{\left(n_{s}\right)} \in \mathbb{R}^{n_{p n_{s}}}$, i.e., $x_{p}=$ $\left(x_{p}^{(1)}, x_{p}^{(2)}, \ldots, x_{p}^{\left(n_{s}\right)}\right)$ and that the measure of each $x_{p}^{(i)}$ is available only at some time instances $t_{k}^{(i)}, k \in \mathbb{N}$, not known a priori. Let, for each $i \in \mathcal{N}:=\left\{1,2, \ldots, n_{s}\right\}, M_{i} \subset \mathbb{R}_{\geq 0}$ be the set of measurement times of $x_{p}^{(i)}$, i.e., $M_{i}=\left\{t_{k}^{(i)}\right\}_{k=0}^{\infty}$, let $\mathbb{M}=\bigcup_{i=1}^{n_{s}} M_{i}$ the set of all measurement times, and let $K \in \mathbb{R}^{n_{u} \times n_{p}}$ be a given control gain. Moreover, suppose that the arrival of a new measurement can be instantaneously detected and that the plant model is known ${ }^{1}$. Then, inspired by [12], we want to study the stability of the closed-loop system obtained by interconnecting the following controller with state jumps to the plant (1)

$$
\mathcal{K}\left\{\begin{array}{clrl}
\dot{\eta}(t) & =A \eta(t)+B \operatorname{sat}(K \eta(t)) & & t \notin \mathbb{M} \\
\eta^{(i)}\left(t^{+}\right) & =x_{p}^{(i)}(t) & & t \in M_{i}, i \in \mathcal{N} \\
\zeta(t) & =K \eta & & t \in \mathbb{R}_{\geq 0}
\end{array}\right.
$$

In particular, $\eta \in \mathbb{R}^{n_{p}}$ is the controller state, $\zeta \in \mathbb{R}^{n_{u}}$ is the controller output and the interconnection of the controller and the plant is realized by setting $v=\zeta$.

The operating principle of the controller in (3) is as follows. The arrival of a new measurement $x_{p}^{(i)}$ for some $i \in \mathcal{N}$ triggers an instantaneous jump in the controller state. Specifically, at each jump, the measured vector $x_{p}^{(i)}$ is instantaneously stored in $\eta^{(i)}$. If different components of $x_{p}$ are measured simultaneously, then all the corresponding components of $\eta$ get updated accordingly. Then, in between consecutive measurements, $\eta$ is continuously updated according to continuous-time dynamics so to mimic the plant dynamics and its value is continuously used in place of the plant state $x_{p}^{(i)}$ in a static state-feedback controller scheme. It appears clear that, as long as the sampling events occur sufficiently often, the controller state $\eta$ provides an accurate estimate of the plant state, which in turn suggests that closedloop stability should be preserved. In particular, we consider the following assumption on the event times:

Assumption 1: For each $i \in \mathcal{N}$, the sequence $\left\{t_{k}^{(i)}\right\}_{k=1}^{\infty}$ is strictly increasing and unbounded and there exist two positive real scalars $T_{1}^{(i)} \leq T_{2}^{(i)}$ such that

$$
\begin{aligned}
& 0 \leq t_{1}^{(i)} \leq T_{2}^{(i)} \\
& T_{1}^{(i)} \leq t_{k+1}^{(i)}-t_{k}^{(i)} \leq T_{2}^{(i)} \quad \forall k \in \mathbb{N}
\end{aligned}
$$

Remark 1: The lower bound in condition (4) prevents the existence of accumulation points in the sequences $\left\{t_{k}^{(i)}\right\}_{k=1}^{\infty}$ and hence avoids the existence of Zeno behaviors, which are typically undesired in practice. In fact, for each $i=$ $1,2, \ldots, n_{p}, T_{1}^{(i)}$ defines a strictly positive minimum time in between consecutive measurements of $x_{p}^{(i)}$. Furthermore, for each $i=1,2, \ldots, n_{p}, T_{2}^{(i)}$ defines the maximum time in between consecutive measurements of $x_{p}^{(i)}$.

Remark 2: Due to asynchronous measurements of the plant state components, as opposed to [12], one cannot assume the controller state to converge in finite-time to the plant state and this renders the analysis of the closed-loop system more challenging. Nonetheless, it is worthwhile to observe that for each initial condition $\left(x_{0}, \eta_{0}\right)$ such that

\footnotetext{
${ }^{1}$ Although our construction relies on the model of the plant, closedloop asymptotic stability is preserved in the presence of small parametric uncertainties. Due to space constraints, robustness issues are not discussed in this paper and will be presented elsewhere.
} 
$x_{0}=\eta_{0}$, the resulting trajectories $\left(\phi_{p}, \phi_{c}\right)$ of the closedloop system are such that $\phi_{p}=\phi_{c}$. Thus, for such initial conditions the effect of the sampling is totally compensated by the controller. On the other hand, in real-world settings, the (unavoidable) presence of plant uncertainties renders the occurrence of such a behavior quite unlikely.

By following a similar approach as in [15], our goal is to provide sufficient conditions for local asymptotic stability for the closed-loop system obtained by interconnecting controller (3) with plant (1). More precisely, the problem we solve is as follows:

Problem 1: Given the closed-loop system obtained by interconnecting controller (3) with the plant (1) via $v=\zeta$, provide an estimate of the basin of attraction for the origin of the closed-loop system.

By interconnecting controller (3) with plant (1) via $v=\zeta$, by defining $\tilde{\eta}:=x_{p}-\eta$, and by denoting $x_{c l}=\left(x_{p}, \tilde{\eta}\right)$, the closed-loop system is modeled by the following dynamical system with jumps:

$$
\begin{cases}\dot{x}_{c l}(t)=A_{c l} x_{c l}(t)+B_{c l} \mathrm{dz}\left(K_{c l} x_{c l}(t)\right) & t \notin \mathbb{M} \\
x_{c l}\left(t^{+}\right)=\left(\begin{array}{cc}
\mathbf{I} & \mathbf{0} \\
\mathcal{X}_{\mathbb{M}}(t) & \mathbf{0}
\end{array}\right) x_{c l}(t) & t \in \mathbb{M}\end{cases}
$$

where

$$
\begin{aligned}
& A_{c l}:=\left(\begin{array}{cc}
A+B K & -B K \\
\mathbf{0} & A
\end{array}\right) \\
& B_{c l}:=\left(\begin{array}{c}
B \\
\mathbf{0}
\end{array}\right), K_{c l}:=\left(\begin{array}{ll}
K & -K
\end{array}\right) \\
& \mathbb{M} \ni t \mapsto \mathcal{X}_{\mathbb{M}}(t)=\operatorname{diag}\left\{X_{M_{1}}^{(1)}(t), X_{M_{2}}^{(2)}(t), \ldots, X_{M_{n_{s}}}^{\left(n_{s}\right)}(t)\right\}
\end{aligned}
$$

where for each $t \in \mathbb{M}, i \in \mathcal{N}$

$$
X_{M_{i}}^{(i)}(t)= \begin{cases}\mathbf{0}_{n_{p_{i}} \times n_{p_{i}}} & \text { if } t \in M_{i} \\ \mathbf{I}_{n_{p_{i}}} & \text { elsewhere }\end{cases}
$$

and for each $s \in \mathbb{R}^{n_{u}}, s \mapsto \operatorname{dz}(s):=\operatorname{sat}(s)-s$.

\section{B. Hybrid modeling}

The fact that the closed-loop system experiences jumps when a new measurement is available suggests that the dynamics of the closed-loop system (5) can be described via a hybrid system. We provide a hybrid model in the framework in [13] that captures the behavior due to each possible evolution generated by any sequence $\left\{t_{k}^{(i)}\right\}_{k=1}^{\infty}$, for $i \in \mathcal{N}$, satisfying (4). This approach leads to a model with nonunique solutions allowing one to establish a strong result for all family of sequences $\left\{t_{k}^{(i)}\right\}_{k=1}^{\infty}$, for $i \in \mathcal{N}$, satisfying (4). The proposed approach requires to model the hidden time-driven mechanism triggering the jumps of the controller. To this end, similarly as in [16], we add $n_{s}$ timer variables $\tau_{1}, \tau_{2}, \ldots, \tau_{n_{s}}$ to keep track of the duration of flows and to trigger jumps according to the mechanism in (5). To accomplish that, we make $\tau_{1}, \tau_{2}, \ldots, \tau_{n_{s}}$ decrease as ordinary time $t$ increases and, whenever $\tau_{i}=0$ for some $i \in \mathcal{N}$, reset it to any point in $\left[T_{1}^{(i)}, T_{2}^{(i)}\right]$, so as to enforce
(4). In particular, define $\tau:=\left(\tau_{1}, \tau_{2}, \ldots, \tau_{n_{s}}\right)$

$$
\mathcal{T}:=\chi_{i=1}^{n_{s}}\left[0, T_{2}^{(i)}\right]
$$

and for all $i \in \mathcal{N}, \tau \in \mathcal{T}$

$$
G_{\tau_{i}}(\tau):=\left\{\chi \in \mathbb{R}^{n_{s}}: \chi_{i} \in\left[T_{1}^{(i)}, T_{2}^{(i)}\right], \forall k \in \mathcal{N} \backslash\{i\}, \chi_{k}=\tau_{k}\right\}
$$

Then, we consider the following dynamics for $\tau$

$$
\begin{array}{lll}
\dot{\tau} & =-\mathbf{1}_{n_{s}} & \tau \in \mathcal{T} \\
\tau^{+} \quad \in \bigcup_{i \in\left\{k \in \mathcal{N}: \tau \in \mathcal{D}_{k}\right\}} G_{\tau_{i}}(\tau) & \tau \in \widehat{\mathcal{D}}_{\tau}
\end{array}
$$

where for each $i \in \mathcal{N}$

$$
\mathcal{D}_{i}:=\left\{\tau \in \mathcal{T}: \tau_{i}=0\right\} \quad \widehat{\mathcal{D}}_{\tau}:=\bigcup_{i \in \mathcal{N}} \mathcal{D}_{i}
$$

Let $x:=\left(x_{c l}, \tau\right) \in \mathbb{R}^{2 n_{p}} \times \mathcal{T}$ be the vector state. We define for each $x \in \mathcal{C}:=\mathbb{R}^{2 n_{p}} \times \mathcal{T}$, where $\mathcal{C}$ is the flow set, the flow map as

$$
f(x):=\left(\begin{array}{c}
A_{c l} x_{c l}+B_{c l} \mathrm{dz}\left(K_{c l} x_{c l}\right) \\
-\mathbf{1}_{n_{s}}
\end{array}\right)
$$

For each $i \in \mathcal{N}$ and $\tilde{\eta} \in \mathbb{R}^{n_{p}}$, define

$$
J_{i}(\tilde{\eta})=\operatorname{diag}\left\{E_{1}^{(i)}, E_{2}^{(i)}, \ldots, E_{n_{s}}^{(i)}\right\} \tilde{\eta} \quad \forall i \in \mathcal{N}
$$

where for each $i, j \in \mathcal{N}$

$$
E_{j}^{(i)}= \begin{cases}\mathbf{0}_{n_{p_{j}} \times n_{p_{j}}} & \text { if } i=j \\ \mathbf{I}_{n_{p_{j}}} & \text { elsewhere }\end{cases}
$$

and let for every $(\tilde{\eta}, \tau) \in \mathbb{R}^{n_{p}} \times \widehat{\mathcal{D}}_{\tau}$

$$
\mathcal{J}(\tilde{\eta}, \tau):=\bigcup_{i \in\left\{k \in \mathcal{N}: \tau \in \mathcal{D}_{k}\right\}}\left(J_{i}(\tilde{\eta}) \times G_{\tau_{i}}(\tau)\right)
$$

We define for each $x \in \mathcal{D}:=\mathbb{R}^{2 n_{p}} \times \widehat{\mathcal{D}}_{\tau}$, where $\mathcal{D}$ is the jump set, the jump map as

$$
G(x)=\left(x_{p}, \mathcal{J}(\tilde{\eta}, \tau)\right)
$$

Therefore, the dynamics of the closed-loop system can be represented by following hybrid system

$$
\mathcal{H}_{c l}\left\{\begin{array}{llll}
\dot{x} & = & f(x) & x \in \mathcal{C} \\
x^{+} & \in & G(x) & x \in \mathcal{D}
\end{array}\right.
$$

An important question that needs to be addressed to guarantee the robustness of the closed-loop system with respect to small perturbations, it is whether $\mathcal{H}_{c l}$ is well-posed; see [13] for more details and consequences of well-posedness. A positive answer to this question is given by the following result.

Lemma 1: The hybrid system (5) is well-posed.

Remark 3: Notice that when $\tau \in \mathcal{D}_{i} \cap \mathcal{D}_{j}$ for some $i \neq j \in \mathcal{N}$, then, only one component of the vector $\tau$ undergoes a change and multiple consecutive jumps occur. This phenomenon directly follows from having defined an outer semicontinuous jump map.

Concerning the existence of solutions to system $\mathcal{H}_{c l}$, it is straightforward to check that for every initial condition 
$\phi(0,0) \in \mathcal{C} \cup \mathcal{D}$, the corresponding maximal solution $\phi$ to $\mathcal{H}_{c l}$ exists and is complete, i.e., $\sup \operatorname{dom} \phi=\infty$. Moreover, the definition of the data of $\mathcal{H}_{c l}$, similarly as in [16], ensures that for each solution, at most $n_{s}$ jumps can occur consecutively without flowing. Such a property, along with (4), ensures that for every maximal solutions $\phi$ to $\mathcal{H}_{c l}$ and each $(t, j) \in \operatorname{dom} \phi$ such that $(t, s) \in$ $\operatorname{dom} \phi$ for some $s \in\left\{j+1, j+2, \ldots, j+n_{s}\right\}$, one has $\left(\left[t, t+\min \left\{T_{1}^{(1)}, T_{1}^{(2)}, \ldots, T_{1}^{\left(n_{s}\right)}\right\}\right] \times\{s\}\right) \subset \operatorname{dom} \phi$. Essentially, the domain of the solutions to $\mathcal{H}_{c l}$, manifests an average dwell-time property, with dwell-time $\tau_{D}=$ $\min \left\{T_{1}^{(1)}, T_{1}^{(2)}, \ldots, T_{1}^{\left(n_{s}\right)}\right\}$ and offset $N_{0}=n_{s}$, which for all $(t, j) \in \operatorname{dom} \phi$ implies $\tau_{D} j \leq t+n_{s} \tau_{D}$; see, e.g., [13, Example 2.15]. Such a property imposes a strictly positive uniform lower bound on the length of every flow interval, preventing from the existence of Zeno solutions.

To solve Problem 1, our approach is to determine sufficient conditions to ensure that the following set ${ }^{2}$

$$
\mathcal{A}=\{0\} \times \mathcal{T}
$$

is asymptotically stable for $\mathcal{H}_{c l}$. In particular, in this work, we consider the following notion of asymptotic stability for a compact set:

Definition 1 (Local asymptotic stability [13]): Let $\mathcal{A} \subset$ $\mathbb{R}^{2 n_{p}+n_{s}}$ be compact. The set $\mathcal{A}$ is said to be

- stable for $\mathcal{H}_{c l}$ if for every $\epsilon>0$ there exists $\delta>0$ such that for every $\phi \in \mathcal{S}_{\mathcal{H}_{c l}}(\mathcal{A}+\delta \mathbb{B})$, one has that $|\phi(t, j)|_{\mathcal{A}} \leq \epsilon$ for every $(t, j) \in \operatorname{dom} \phi$

- locally attractive for $\mathcal{H}_{c l}$ if there exists $\mu>0$ such that every maximal solution $\phi$ to $\mathcal{H}_{c l}$ with $|\phi(0,0)|_{\mathcal{A}} \leq \mu$ is complete and such that

$$
\lim _{t+j \rightarrow \infty}|\phi(t, j)|_{\mathcal{A}}=0
$$

- locally asymptotically stable for $\mathcal{H}_{c l}$ if both stable and locally attractive.

Definition 2 (Basin of attraction [13]): Let $\mathcal{A} \subset$ $\mathbb{R}^{2 n_{p}+n_{s}}$ be locally asymptotically stable for $\mathcal{H}_{c l}$. The basin of attraction of $\mathcal{A}$ is the set of points $\mathcal{B}_{\mathcal{A}} \subset \mathbb{R}^{2 n_{p}+n_{s}}$ such that each $\phi \in \mathcal{S}_{\mathcal{H}_{c l}}\left(\mathcal{B}_{\mathcal{A}}\right)$ is complete and converges to $\mathcal{A}$.

In light of the above definitions, it turns out that Problem 1 can be solved by determining the basin of attraction of $\mathcal{A}$ defined in (11). On the other hand, the determination of such a set is a difficult problem and cannot be solved in general. To overcome this drawback, in the following section we provide sufficient conditions in the form of matrix inequalities to determine subsets of the basin of attraction of $\mathcal{A}$. Building on such conditions, we show how semidefinite programming techniques can be used to reduce the conservatism of the proposed estimates.

${ }^{2}$ By the definition of the system $\mathcal{H}_{c l}$ and of the set $\mathcal{A}$, for every $x \in$ $\mathcal{C} \cup \mathcal{D} \cup G(\mathcal{D}),|x|_{\mathcal{A}}=\left|\left(x_{p}, \tilde{\eta}\right)\right|$.

\section{MAIN RESUltS}

\section{A. Sufficient Conditions}

In this section we provide sufficient conditions for the solution to Problem 1. To accomplish this task, we pursue a Lyapunov approach. In particular, we state sufficient conditions in the form of matrix inequalities guaranteeing local asymptotic stability of the set $\mathcal{A}$ in (11) for $\mathcal{H}_{c l}$ and providing an estimate of the basin of attraction of $\mathcal{A}$. The derivation of such a result builds from [13, Corollary 8.4, Corollary 8.9] and on the generalized sector condition for the deadzone nonlinearity in [17].

Theorem 1 (Local asymptotic stability): Let $W \in \mathcal{S}_{+}^{n_{p}}$, $R_{1} \in \mathcal{S}_{+}^{n_{p_{1}}}, R_{2} \in \mathcal{S}_{+}^{n_{p_{2}}}, \ldots, R_{n_{s}} \in \mathcal{S}_{+}^{n_{p_{n_{s}}}}, S \in \mathcal{D}_{+}^{n_{u}}$, $\sigma_{1}, \sigma_{2}, \ldots, \sigma_{n_{s}} \in \mathbb{R}_{>0}, Z, J \in \mathbb{R}^{n_{u} \times n_{p}}$. Assume that

$$
\left(\begin{array}{ccc}
W & \mathbf{0} & Z_{(i)}^{\top} \\
\mathbf{0} & \bigoplus_{j=1}^{n_{s}} R_{j} & J_{(i)}^{\top} \\
\bullet & \bullet & \frac{\bar{v}_{i}^{2}}{\bar{\mu}}
\end{array}\right) \geq \mathbf{0} \quad i=1,2, \ldots, n_{u}
$$

and that for $\operatorname{each}^{3} \tau \in \mathcal{T}$

$$
\left(\begin{array}{ccc}
\operatorname{He}((A+B K) W) & -B K & B S-W K^{\boldsymbol{\top}}-Z^{\top} \\
\bullet & \operatorname{He}(R(\tau) A)-\Sigma R(\tau) & K^{\top}-J^{\top} \\
\bullet & \bullet & -2 S
\end{array}\right)<\mathbf{0}
$$

where $^{4} \Sigma:=\bigoplus_{j=1}^{n_{s}} \sigma_{j} \mathbf{I}_{n_{p_{j}}}$ and for all $\tau \in \mathcal{T}, R(\tau):=$ $\bigoplus_{j=1}^{n_{s}} e^{\sigma_{j} \tau_{j}} R_{j}$. Define ${ }^{5}$

$$
\mathcal{T} \ni \tau \mapsto \widehat{P}(\tau):=W^{-1} \oplus R(\tau)
$$

Then, set $\mathcal{A}$ defined in (11) is locally asymptotically stable for $\mathcal{H}_{c l}$ and the set

$$
\mathcal{E}_{\bar{\mu}}:=\left\{x \in \mathcal{C}: x_{c l}^{\top} \widehat{P}(\tau) x_{c l} \leq \bar{\mu}\right\}
$$

is included in the basin of attraction of $\mathcal{A}$.

\section{B. Numerical Issues}

Theorem 1 turns the solution to Problem 1 into the feasibility problem of some matrix inequalities. On the other hand, the search for feasible solutions to (13) is quite challenging since such conditions need to be checked over the box $\mathcal{T}$. To overcome this problem, next we show that (13) can be turned without any loss into a finite set of matrix inequalities.

Proposition 1: Let $W \in \mathcal{S}_{+}^{n_{p}}, R_{1} \in \mathcal{S}_{+}^{n_{p_{1}}}, R_{2} \in$ $\mathcal{S}_{+}^{n_{p_{2}}}, \ldots, R_{n_{s}} \in \mathcal{S}_{+}^{n_{p_{n_{s}}}}, S \in \mathcal{D}_{+}^{n_{u}}, \sigma_{1}, \sigma_{2}, \ldots, \sigma_{n_{s}} \in \mathbb{R}_{>0}$, and $Z, J \in \mathbb{R}^{n_{u} \times n_{p}}$ be given. The following two items are equivalent ${ }^{6}$

(i) (13) holds

\footnotetext{
${ }^{3}$ There is a missing $S$ in (13) and (14).

${ }^{4}$ The symbol $\Sigma$ is undefined in the published version.

${ }^{5}$ In the published version, the first definition of the symbol $\widehat{P}(\tau)$ appears in Corollary 1.

${ }^{6}$ There is a missing $\mathbf{I}$ in the set defined in equation (14).
} 
(ii) let $Q:=\bigoplus_{i=1}^{n_{s}} R_{i}$

$$
\begin{aligned}
& \mathcal{N}(\Psi):=\left(\begin{array}{ccc}
\operatorname{He}((A+B K) W) & -B K & B S-W K^{\top}-Z^{\top} \\
\bullet & \operatorname{He}(Q \Psi A)-\Sigma Q \Psi & K^{\top}-J^{\top} \\
\bullet & \bullet & -2 S
\end{array}\right)<\mathbf{0} \\
& \forall \Psi \in\left\{\bigoplus_{i=1}^{n_{s}} \psi_{i} \mathbf{I}_{n_{i}}: \psi_{i} \in\left\{1, e^{\sigma_{i} T_{2}^{(i)}}\right\}\right\}
\end{aligned}
$$

Another issue that needs to be tackled in Theorem 1 is related to the fact that the resulting estimate of the basin of attraction is a subset of $\mathcal{C}$, hence intrinsically dependent on the "artificial" variable $\tau$. Indeed, from a practical standpoint, one is interested in dealing with sets of initial conditions for the closed-loop system (5). The result given next allows one to achieve this goal.

Corollary 1: Assume that there exist $W \in \mathcal{S}_{+}^{n_{p}}, R_{1} \in$ $\mathcal{S}_{+}^{n_{p_{1}}}, R_{2} \in \mathcal{S}_{+}^{n_{p_{2}}}, \ldots, R_{n_{s}} \in \mathcal{S}_{+}^{n_{p_{n_{s}}}}, S \in \mathcal{D}_{+}^{n_{u}}$, $\sigma_{1}, \sigma_{2}, \ldots, \sigma_{n_{s}} \in \mathbb{R}_{>0}$, and $Z, J \in \mathbb{R}^{n_{u} \times n_{p}}$ such that (12)(13) hold. Let

$$
\mathcal{T} \ni \tau \mapsto \widehat{P}(\tau):=W^{-1} \oplus R(\tau)
$$

and $^{7}$

$$
\tau^{\star}:=\left(T_{2}^{(1)}, T_{2}^{(2)}, \ldots, T_{2}^{\left(n_{s}\right)}\right)
$$

Then, the set $\mathcal{E}\left(\widehat{P}\left(\tau^{\star}\right), \bar{\mu}\right) \times \mathcal{T}$ is included in the basin of attraction of $\mathcal{A}$ for (10).

Remark 4: Having an estimate of the basin of attraction that is " $\tau$-independent" allows one to define convenient size criteria that can be conveniently exploited in an optimization setup to optimize the size of the resulting set. This aspect is shown in the subsequent section.

Remark 5: It is interesting to notice that the "size" of the estimate of the basin of attraction provided by Corollary 1 depends on the vector $\tau^{\star}$. In particular, one can remark that as $\tau^{\star}$ goes to infinity, $\mathcal{E}\left(\widehat{P}\left(\tau^{\star}\right), \bar{\mu}\right)$ approaches the origin while as $\tau^{\star}$ goes to zero, $\mathcal{E}\left(\widehat{P}\left(\tau^{\star}\right), \bar{\mu}\right)$ approaches $\mathcal{E}(\widehat{P}(0), \bar{\mu})$. Thus, on the one hand, $\mathcal{E}(\widehat{P}(0), \bar{\mu})$ provides the largest estimate of the basin of attraction achievable via our methodology, on the other hand, increasing $\tau^{\star}$ tends to give rise to smaller estimates of the basin of attraction.

\section{Optimization Issues}

With the objective of reducing the conservatism of the proposed analysis, Problem 1 can be formalized as a suitable optimization problem. In particular, similarly as in [18], [7], [15], the following optimization problem can be considered:

Problem 2 (Estimation of the basin of attraction): "Maximize" the set $\mathcal{E}\left(\widehat{P}\left(\tau^{\star}\right), \bar{\mu}\right)$.

To properly formulate the above optimization problem, one needs to define an adequate measure for the ellipsoidal set $\mathcal{E}\left(\widehat{P}\left(\tau^{\star}\right), \bar{\mu}\right)$. To this aim, different criteria are proposed in the literature; see [19]. In this work, we consider $\operatorname{trace}\left(\frac{1}{\bar{\mu}} \widehat{P}\left(\tau^{\star}\right)\right)=\frac{1}{\bar{\mu}} \operatorname{trace}\left(W^{-1}+R\left(\tau^{\star}\right)\right)$ as a size criterion. In particular, larger values of $\operatorname{trace}\left(\frac{1}{\bar{\mu}} \widehat{P}\left(\tau^{\star}\right)\right)$ are associated to a smaller ellipsoid. Therefore, the objective consists of

\footnotetext{
${ }^{7}$ The symbol $\tau^{\star}$ is undefined in the conference version.
}

minimizing $\frac{1}{\bar{\mu}} \operatorname{trace}\left(W^{-1}+R\left(\tau^{\star}\right)\right)$. On other hand, to avoid dealing in the solution to the resulting optimization problems with $W^{-1}$ as a decision variable, one can consider the following additional constraint

$$
\left(\begin{array}{cc}
M_{W} & \mathbf{I} \\
\bullet & W
\end{array}\right) \geq \mathbf{0}
$$

which is equivalent to $M_{W}-W^{-1} \geq \mathbf{0}$. That allows one to achieve the minimization of the considered objective by minimizing the objective $\frac{1}{\bar{\mu}} \operatorname{trace}\left(M_{W}+R\left(\tau^{\star}\right)\right)$. By setting $\bar{\mu}^{-1}:=\delta$, this leads to the following formulation for Problem 2

$$
\left\{\begin{array}{l}
\operatorname{minimize} \omega_{1} \delta+\omega_{2} \operatorname{trace}\left(M_{W}+R\left(\tau^{\star}\right)\right) \\
\text { s.t. } \\
W>\mathbf{0}, Q>\mathbf{0},(14),(15), S \in \mathcal{D}_{+}^{n_{u}} \\
\left(\begin{array}{ccc}
W & \mathbf{0} & Z_{(i)}^{\top} \\
\mathbf{0} & \bigoplus_{j=1}^{n_{s}} R_{j} & J_{(i)}^{\top} \\
\bullet & \bullet & \delta \bar{v}_{i}^{2}
\end{array}\right) \geq \mathbf{0} \quad i=1,2, \ldots, n_{u}
\end{array}\right.
$$

where $\omega_{1}, \omega_{2} \in \mathbb{R}_{>0}$ are tuning parameters.

Whenever the scalars $\sigma_{1}, \sigma_{2}, \ldots, \sigma_{n_{s}}$ are selected, the above optimization problem is a semidefinite program, i.e., an optimization problem with linear objective and linear matrix inequality constraints. Therefore, the solution to this problem can be efficiently obtained via numerical available software; see [19]. On the other hand, the nonlinearities involving the real numbers $\sigma_{i}$, for $i=1,2, \ldots, n_{s}$, are easily manageable (at least when $n_{s}$ is small enough) in a numerical scheme by treating these scalars as tuning parameters or by selecting those via an iterative search.

\section{NUMERICAL EXAMPLE}

Consider the example in [20], which is defined by the following data:

$$
\begin{aligned}
& A=\left(\begin{array}{cc}
-0.8 & -0.01 \\
1 & 0.1
\end{array}\right), B=\left(\begin{array}{ll}
0.4 & 0.1
\end{array}\right)^{\top} \\
& K=\left(\begin{array}{ll}
-1.2625 & -1.2679
\end{array}\right)
\end{aligned}
$$

assume the control input is saturated with saturation level $\bar{u}=1$, and set $T_{1}^{(1)}=T_{1}^{(2)}=0.1, T_{2}^{(1)}=0.3, T_{2}^{(2)}=$ $2.35 \times T_{2}^{(1)}$. By solving Problem 2 via a grid search for the scalars $\sigma_{1}, \sigma_{2}$, one gets ${ }^{8}$ :

$W^{-1}=\left(\begin{array}{ll}0.0779 & 0.0629 \\ 0.0629 & 0.0558\end{array}\right), R_{1}=0.041342, R_{2}=0.018985$

$\sigma_{1}=1.8, \sigma_{2}=2.3, \bar{\mu}=2.1380$

To better assess the conservatism of the estimate of the basin of attraction (given by $\mathcal{E}\left(\widehat{P}\left(\tau^{\star}\right), \bar{\mu}\right) \times \mathcal{T}$ ), we pick initial conditions for $\mathcal{H}_{c l}$ in $\mathcal{U}:=\left(\mathcal{E}\left(\widehat{P}\left(\tau^{\star}\right), \bar{\mu}\right) \cap \mathcal{Y}\right) \times \mathcal{T}$, were $\mathcal{Y}:=\left\{\left(x_{p}, \tilde{\eta}\right) \in \mathbb{R}^{2 n_{p}}: \tilde{\eta}=x_{p}\right\}$, i.e., we enforce zero initial conditions for the controller (3). The projection of $\mathcal{U}$ onto $\mathbb{R}^{2}$ (the plant state space) is shown in Fig. 1

\footnotetext{
${ }^{8}$ Numerical solutions to SDP problems are obtained in Matlab ${ }^{\circledR}$ via Mosek [21] thanks to YALMIP [22]. Simulations of hybrid systems are performed in Matlab ${ }^{\circledR}$ via the Hybrid Equations (HyEQ) Toolbox [23]. Code at https://github.com/HybridSystemsLab/IntermittentCtrlSatMrate
} 


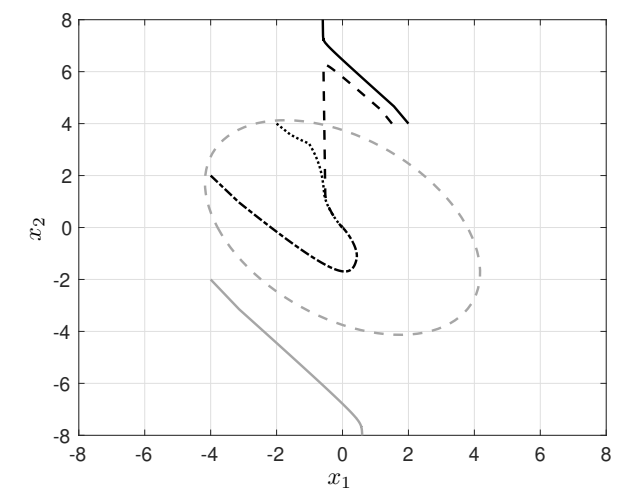

Fig. 1: The set $\mathcal{U}$ projected onto $\mathbb{R}^{2}$ (dashed gray) and some trajectories of the plant state: $x_{0}=(-4,2)$ (dash-dotted black), $x_{0}=(-2,4)$ (black dotted), $x_{0}=(2,4)$ (solid black), $x_{0}=(-2,-4)$ (gray solid), and $x_{0}=(1.5,4)$ (dashed black).

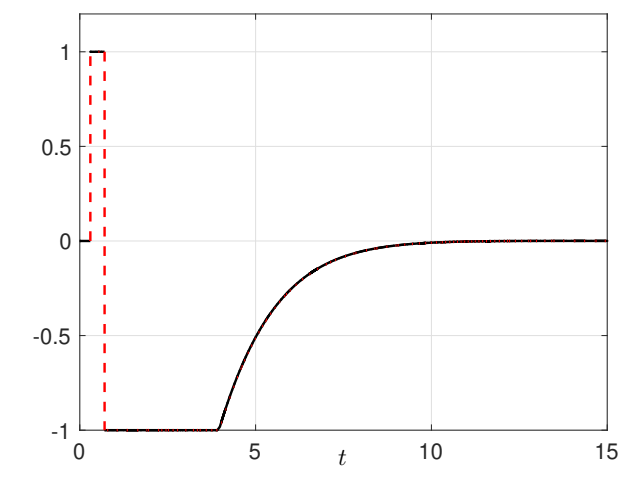

Fig. 2: The evolution of the control input $u$ from $x_{0}=(-2,4)$.

along with some trajectories for the plant state. In particular, let $\phi=\left(\phi_{x_{p}}, \phi_{\tilde{\eta}}, \phi_{\tau_{1}}, \phi_{\tau_{2}}\right)$ be a solution to $\mathcal{H}_{c l}$, in this simulation we selected initial conditions of the form $\phi(0,0)=\left(x_{0}, x_{0}, T_{2}^{(1)}, T_{2}^{(2)}\right), x_{0} \in \mathbb{R}^{2}$, and for each $\left(t_{j}, j+1\right) \in \operatorname{dom} \phi$

$$
\begin{aligned}
& \phi_{\tau_{1}}\left(t_{j}, j+1\right)=\frac{T_{2}^{(1)}-T_{1}^{(1)}}{2} \sin \left(t_{j}\right)+\frac{T_{2}^{(1)}+T_{1}^{(1)}}{2} \\
& \phi_{\tau_{1}}\left(t_{j}, j+1\right)=\frac{T_{2}^{(2)}-T_{1}^{(2)}}{2} \sin \left(10 t_{j}\right)+\frac{T_{2}^{(2)}+T_{1}^{(2)}}{2}
\end{aligned}
$$

In particular, observe that

$$
\left(x_{0}, x_{0}, T_{2}^{(1)}, T_{2}^{(2)}\right) \in \mathcal{E}\left(\widehat{P}\left(\tau^{\star}\right), \bar{\mu}\right) \times \mathcal{T}
$$

if and only if $x_{0} \in \Pi_{\mathbb{R}^{2}}(\mathcal{U})$.

Fig.1 points out that for some initial conditions outside the set $\mathcal{E}\left(\widehat{P}\left(\tau^{\star}\right), \bar{\mu}\right) \times \mathcal{T}$ the plant state diverges. On the other hand, the conservatism of our approach is revealed by the existence of converging trajectories from points outside $\mathcal{E}\left(\widehat{P}\left(\tau^{\star}\right), \bar{\mu}\right) \times \mathcal{T}\left(x_{0}=(1.5,4)\right)$. In Fig. 2, we reported the evolution of the control input when $x_{0}=(-2,4)$. The figure emphasizes that, as foreseen through our methodology, despite the saturation of the control input, asymptotic stability is preserved.

\section{CONCLUSion}

Regional stability analysis of linear systems with multirate samplers and actuator saturation was studied. The approach we proposed is able to provide explicit estimates for the basin of attraction by the use of semidefinite programming.

The results presented in this paper open the door to several research directions. In particular, the extension of these results to $\mathcal{L}_{2}$-external stability is currently an ongoing work. Other valuable extensions of this work pertain to the design of state estimators to address more realistic scenarios in which the vector state is not fully measurable and the analysis of the effect of plant uncertainties.

\section{REFERENCES}

[1] L. Hetel, C. Fiter, H. Omran, A. Seuret, E. Fridman, J.-P. Richard, and S. I. Niculescu, "Recent developments on the stability of systems with aperiodic sampling: An overview," Automatica, vol. 76, pp. 309-335, 2017.

[2] D. Nešić, A. R. Teel, and D. Carnevale, "Explicit computation of the sampling period in emulation of controllers for nonlinear sampled-data systems," IEEE transactions on Automatic Control, vol. 54, no. 3, pp. 619-624, 2009.

[3] E. Fridman, A. Seuret, and J.-P. Richard, "Robust sampled-data stabilization of linear systems: an input delay approach," Automatica, vol. 40, no. 8, pp. 1441-1446, 2004.

[4] M. Moarref, C. Ossa-Gomez, and L. Rodrigues, "Multiloop controller design for a fly-by-wireless uav quadrotor based on a multirate sampled-data model," IEEE Transactions on Aerospace and Electronic Systems, vol. 51, no. 3, pp. 2319-2331, 2015.

[5] S. Lall and G. Dullerud, "An LMI solution to the robust synthesis problem for multi-rate sampled-data systems," Automatica, vol. 37, no. 12, pp. 1909-1922, 2001.

[6] J.-Y. Yen, Y.-L. Chen, and M. Tomizuka, "Variable sampling rate controller design for brushless dc motor," in Proceedings of the 41st IEEE Conference on Decision and Control, 2002, vol. 1. IEEE, 2002, pp. 462-467.

[7] S. Tarbouriech, G. Garcia, J. M. Gomes da Silva Jr., and I. Queinnec, Stability and stabilization of linear systems with saturating actuators. Springer, 2011.

[8] L. Zaccarian and A. R. Teel, Modern anti-windup synthesis: control augmentation for actuator saturation. Princeton University Press, 2011.

[9] D. Dai, T. Hu, A. R. Teel, and L. Zaccarian, "Output feedback synthesis for sampled-data system with input saturation," in American Control Conference (ACC), 2010. IEEE, 2010, pp. 1797-1802.

[10] A. Seuret and J. M. Gomes da Silva Jr., "Taking into account period variations and actuator saturation in sampled-data systems," Systems \& Control Letters, vol. 61, no. 12, pp. 1286-1293, 2012.

[11] J. M. Gomes da Silva Jr., I. Queinnec, A. Seuret, and S. Tarbouriech, "Regional stability analysis of discrete-time dynamic output feedback under aperiodic sampling and input saturation," IEEE Transactions on Automatic Control, vol. 61, no. 12, pp. 4176-4182, 2016.

[12] L. A. Montestruque and P. J. Antsaklis, "Stability of model-based networked control systems with time-varying transmission times," IEEE Transactions on Automatic Control, vol. 49, no. 9, pp. 15621572, 2004.

[13] R. Goebel, R. G. Sanfelice, and A. R. Teel, Hybrid Dynamical Systems: Modeling, Stability, and Robustness. Princeton University Press, 2012.

[14] M. Moarref and L. Rodrigues, "Sensor allocation with guaranteed exponential stability for linear multi-rate sampled-data systems," International Journal of Robust and Nonlinear Control, vol. 26, no. 7, pp. 1512-1529, 2016.

[15] T. Hu, A. R. Teel, and L. Zaccarian, "Stability and performance for saturated systems via quadratic and nonquadratic Lyapunov functions," IEEE Transactions on Automatic Control, vol. 51, no. 11, pp. 17701786, 2006. 
[16] F. Ferrante, F. Gouaisbaut, R. G. Sanfelice, and S. Tarbouriech, "Observer-based control design for linear systems in the presence of limited measurement streams and intermittent input access," in Proceedings of the 2015 American Control Conference (ACC), 2015, pp. 4689-4694.

[17] S. Tarbouriech, C. Prieur, and J. M. Gomes da Silva Jr., "Stability analysis and stabilization of systems presenting nested saturations," IEEE Transactions on Automatic Control, vol. 51, no. 8, pp. 13641371, 2006.

[18] T. Hu, A. R. Teel, and L. Zaccarian, "Anti-windup synthesis for linear control systems with input saturation: Achieving regional, nonlinear performance," Automatica, vol. 44, no. 2, pp. 512-519, 2008.

[19] S. Boyd, L. E. Ghaoui, E. Feron, and V. Balakrishnan, Linear Matrix Inequalities in System and Control Theory. Society for Industrial and
Applied Mathematics, Jun. 1997.

[20] L.-S. Hu, T. Bai, P. Shi, and Z. Wu, "Sampled-data control of networked linear control systems," Automatica, vol. 43, no. 5, pp. 903-911, 2007.

[21] M. ApS, The MOSEK optimization toolbox for MATLAB manual. Version 7.1 (Revision 28)., 2015. [Online]. Available: http://docs. mosek.com/7.1/toolbox/index.html

[22] J. Lofberg, "Yalmip: A toolbox for modeling and optimization in matlab," in Computer Aided Control Systems Design, 2004 IEEE International Symposium on. IEEE, 2004, pp. 284-289.

[23] R. G. Sanfelice, D. Copp, and P. Nanez, "A toolbox for simulation of hybrid systems in matlab/simulink: Hybrid equations (HyEQ) toolbox," in Proceedings of the 16th international conference on Hybrid systems: computation and control. ACM, 2013, pp. 101-106. 